\title{
INFRAVERMELHO NA CARACTERIZAÇÃO DE ÁCIDOS HÚMICOS DE LATOSSOLO SOB EFEITO DE USO CONTÍNUO DE LODO DE ESGOTO(1)
}

\author{
Bruno de Oliveira Dias ${ }^{(2)}$, Carlos Alberto Silva ${ }^{(3)}$, Emanuelle Mercês \\ Barros Soares $^{(4)}$, Wagner Bettiol ${ }^{(5)}$, Mário César Guerreiro ${ }^{(6)} \&$ \\ Maísa Honório Belizário( ${ }^{(7)}$
}

\begin{abstract}
RESUMO
Objetivou-se caracterizar, por meio de espectroscopia no infravermelho com transformada de Fourier (IVTF), possíveis mudanças na natureza química de ácidos húmicos (AHs) extraídos de amostras de Latossolo (0-10 cm) do Campo Experimental da Embrapa Meio Ambiente, em Jaguariúna (SP). Os dados foram obtidos após seis cultivos de milho e a aplicação de doses variáveis de lodo de esgoto de Barueri (LB) (base seca), conforme os tratamentos avaliados: LB0, LB1, LB2, LB4 e LB8, sendo 0, 1, 2, 4 e 8 vezes a necessidade de $\mathrm{N}$ para o milho. Como referência, foi amostrada uma área sob mata. Foram obtidos espectros de ácidos húmicos e do lodo de esgoto utilizado no experimento, e calculados os índices de hidrofobicidade (IH) e de condensação (IC) nas amostras de ácidos húmicos. Foram notadas pequenas alterações na natureza química de AHs de áreas tratadas com lodo de esgoto, em relação à testemunha. Os AHs apresentam grupos aromáticos, -OH fenólicos, $-\mathrm{COOH}$, estruturas alifáticas, carboidratos e impurezas minerais. $\mathrm{O}$ uso contínuo de elevadas doses de LB reduziu a concentração de C-alifático e o grau de condensação dos ácidos húmicos, o que sinaliza que há frações orgânicas
\end{abstract}

\footnotetext{
(1) Parte da Dissertação de Mestrado do primeiro autor, apresentada ao Curso de Pós-Graduação em Ciência do Solo, Departamento de Ciência do Solo, Universidade Federal de Lavras - UFLA. Recebido para publicação em abril de 2006 e aprovado em maio de 2009.

${ }^{(2)}$ Doutorando em Ciência do Solo, Universidade Federal de Lavras - UFLA. Caixa Postal 3037, CEP 37200-000 Lavras (MG). E-mail: b2dias@yahoo.com.br

(3) Professor do Departamento de Ciência do Solo, UFLA. E-mail: csilva@ufla.br

(4) Doutoranda em Agronomia, Solos e Nutrição de Plantas, DPS/UFV. E-mail: mercessoares@yahoo.com.br

(5) Pesquisador da Embrapa Meio Ambiente. Rod. SP 340, Km 127,5. Caixa Postal 69, CEP 13820-000 Jaguariúna (SP). Bolsista do CNPq. E-mail: bettiol@cnpma.embrapa.br

(6) Professor do Departamento de Química, UFLA. E-mail: guerrero@ufla.br

(7) Doutoranda em Solos e Nutrição de Plantas, Escola Superior de Agricultura "Luiz de Queiroz" - ESALQ/USP. Caixa Postal 9, Av. Pádua Dias 11, CEP 13418-900 Piracicaba (SP). E-mail: maisaagro@yahoo.com.br
} 
com maior biodisponibilidade no solo. Na área de mata, o material húmico se caracteriza pelo caráter hidrofóbico e pelo baixo grau de condensação.

Termos para indexação: matéria orgânica, biodisponibilidade, índice de hidrofobicidade, substâncias húmicas.

\title{
SUMMARY: INFRARED SPECTROSCOPY IN THE CHARACTERIZATION OF HUMIC ACIDS FROM AN OXISOL UNDER THE EFFECT OF CONTINUOUS USE OF SEWAGE SLUDGE
}

\begin{abstract}
This study was carried out to characterize the possible changes in the chemical nature of humic acids extracted from an Oxisol $(0-10 \mathrm{~cm})$ by Fourier transform infrared spectroscopy (FTIR), in the experimental area of Embrapa Meio Ambiente, in Jaguariúna (SP), Brazil. The data were obtained after six corn cultivations, and application of variable doses of sewage sludge (dry basis) from Barueri (BSS), in the treatments: BSSO - control without sewage sludge application; NPK - mineral fertilization as required by corn; application of BSS to supply one (BSS1), two (BSS2), for four (BSS4), and eight (BSS8) times the $N$ required by corn. As a reference, a forest area close to the experiment was also sampled. FTIR spectra of humic acids (HA) and the sewage sludge used in the experiments were obtained, and the hydrophobicity (HI) and condensation (CI) indexes for the HA samples were calculated. Small changes in the chemical nature of HA from soil treated with BSS were observed in comparison to that from the control soil. The HA analyzed contained aromatic, phenolics, and carboxylic groups, aliphatic structures, carbohydrates and mineral impurities. The continuous use of high BSS doses reduced the aliphatic-C and condensation degree of $H A$ in the soil, which indicates the presence of organic materials with higher bioavailability in these areas. In the area under forest, the HA fraction was characterized by the hydrophobic character and low condensation degree.
\end{abstract}

Index terms: organic matter, bioavailability, hydrophobicity index, humic substances.

\section{INTRODUÇÃO}

O uso agrícola do lodo de esgoto como fonte de nutrientes para as plantas e seus efeitos sobre as propriedades dos solos já foram verificados em vários estudos (Oliveira et al., 2002; Simonete et al., 2003; Rocha et al., 2004), no entanto, permanecem algumas dúvidas sobre o efeito desse resíduo sobre a composição química da matéria orgânica do solo. Segundo Senesi et al. (1996), em áreas adubadas com lodo de esgoto, torna-se necessário o estudo da composição química da matéria orgânica e, principalmente, das substâncias húmicas presentes nesse resíduo, já que as frações húmicas são indicadores da maturação e da estabilidade do lodo de esgoto. De acordo com Rovira et al. (2002), é preciso investigar também o efeito de doses de lodo de esgoto sobre a natureza química de substâncias húmicas extraídas de solos tratados com o resíduo.

Nos últimos anos, o advento e o aperfeiçoamento das técnicas espectroscópicas para o estudo da matéria orgânica vêm demonstrando o forte potencial da espectroscopia no infravermelho (IV) em auxiliar no entendimento dos processos relacionados com a dinâmica da matéria orgânica após a aplicação de resíduos no solo (Canellas et al., 2000; Rovira et al., 2002). Essa técnica permite analisar a natureza química, a reatividade e o arranjo estrutural de grupos funcionais contendo $\mathrm{O}$ e a eficiência do processo de purificação da amostra quanto há contaminantes como argila, sais e metais (Stevenson, 1982). Em relação às outras técnicas espectroscópicas de análise da matéria orgânica do solo e de seus compartimentos, a análise em IV possibilita avaliar as ligações químicas com momento dipolo grande, ou seja, grupamentos que contêm $\mathrm{O}$. Além disso, na região conhecida como "fingerprint", nenhum composto ou substância apresenta o mesmo espectro entre $1.400-800 \mathrm{~cm}^{-1}$, o que possibilita o estudo da origem, de transformações e de contaminação dos compostos analisados (Canellas, 2008 - comunicação pessoal).

A análise da natureza química dos ácidos húmicos se presta a avaliar a qualidade da matéria orgânica do solo, por se tratar da fração principal das substâncias húmicas e em razão da fração de ácido húmico regular vários processos do solo (Labrador, 1996; Canellas et al., 2000; Rovira et al., 2002). Isso ocorre em função de o comportamento espectroscópico 
de ácidos fúlvicos ser semelhante ao dos polissacarídeos (que representam, em média, somente $25 \%$ do $\mathrm{C}$ total do solo), e de o $\mathrm{C}$ associado à humina não ser representativo da matéria orgânica do solo. Essa fração húmica sofre pequenas modificações, em razão de sua associação com a fração mineral do solo e devido à sua característica apolar (Zech et al., 1997), embora o estudo de Song et al. (2008) revele que há nessa fração componentes oriundos de plantas e microrganismos com diferentes graus de reatividade, como carboidratos, peptídeos, materiais derivados de lignina, lipoproteínas, ácidos gordurosos, material cuticular e peptídeoglicanos.

Analisando as propriedades espectroscópicas de ácidos húmicos, Rovira et al. (2002) verificaram que os materiais húmicos oriundos de solos adubados com lodo de esgoto apresentavam um caráter predominantemente alifático, baixo teor de grupos funcionais oxigenados, altos teores de polissacarídeos contendo $\mathrm{N}$ e $\mathrm{S}$, poucos radicais livres, alta heterogeneidade molecular, baixa polimerização e grau de humificação, em relação à natureza química de ácidos húmicos oriundos de solos não adubados. De acordo com esses autores, as maiores modificações na composição química de ácidos húmicos foram notadas nos materiais oriundos de solos adubados com doses elevadas de esgoto líquido. No estudo de Canellas et al. (2000), os ácidos húmicos extraídos de lodo de esgoto apresentaram maior quantidade de grupos aromáticos e de radicais carboxílicos do que os extraídos de composto de lixo, e as duas frações húmicas analisadas apresentaram natureza química similar à de ácidos húmicos extraídos de solo. De acordo com Rovira et al. (2002), nesses tipos de estudos, o conhecimento da natureza química dos ácidos húmicos torna-se importante por permitir avaliar a eficiência agronômica e o impacto ambiental da aplicação de lodo de esgoto.

Os espectros de infravermelho fornecem informações qualitativas, mas é possível obter determinações semiquantitativas de grupos funcionais, principalmente os que contêm $\mathrm{O}$. Índices relacionados com a hidrofobicidade (IH) são obtidos por meio da relação entre grupos polares e mais apolares; os grupamentos $-\mathrm{CH}_{3}$ em cadeias alquílicas são, caracteristicamente, mais recalcitrantes, enquanto os grupamentos C-O de polissacarídeos são mais suscetíveis à oxidação. Dessa forma, o IH de moléculas orgânicas fornece uma idéia de sua resistência à degradação (Freixo, 2000).

O objetivo deste estudo foi avaliar a natureza química de ácidos húmicos extraídos de Latossolo Vermelho distroférrico sob aplicações sucessivas de doses crescentes de lodo de esgoto, utilizando a técnica de espectroscopia no infravermelho com transformada de Fourier.

\section{MATERIAL E MÉTODOS}

Neste estudo, foram utilizadas amostras da camada superficial $(0-10 \mathrm{~cm})$ de um Latossolo Vermelho distroférrico de textura argilosa (Quadro 1), coletadas no Campo Experimental da Embrapa Meio Ambiente, localizado em Jaguariúna (SP), na latitude de $22^{\circ} 41^{\prime}$ Sul, longitude $47^{\circ} \mathrm{W}$ Gr. e altitude de $570 \mathrm{~m}$.

O solo foi tratado com crescentes doses de lodo de esgoto oriundo da Estação de Tratamento de Esgoto da SABESP, em Barueri, SP (Lodo de Barueri - LB). $\mathrm{Na}$ área em estudo, já haviam sido realizados seis cultivos sucessivos com milho e aplicadas doses variáveis de lodo de esgoto. No quadro 2 são apresentadas as doses aplicadas no experimento por tratamento e por cultivo e, no quadro 3, algumas características químicas do lodo.

O experimento foi constituído pelos seguintes tratamentos: (LB0) testemunha sem adição de lodo de Barueri; (NPK) fertilização mineral indicada para a cultura do milho. As doses de lodo de esgoto foram definidas de acordo com os teores de $\mathrm{N}$ nesse resíduo e com base na necessidade de $\mathrm{N}$ do milho. No tratamento LB1, a aplicação de lodo de esgoto foi realizada visando

Quadro 1. Caracterização química das amostras de solos utilizadas no experimento, coletadas após a sexta aplicação de lodo de esgoto

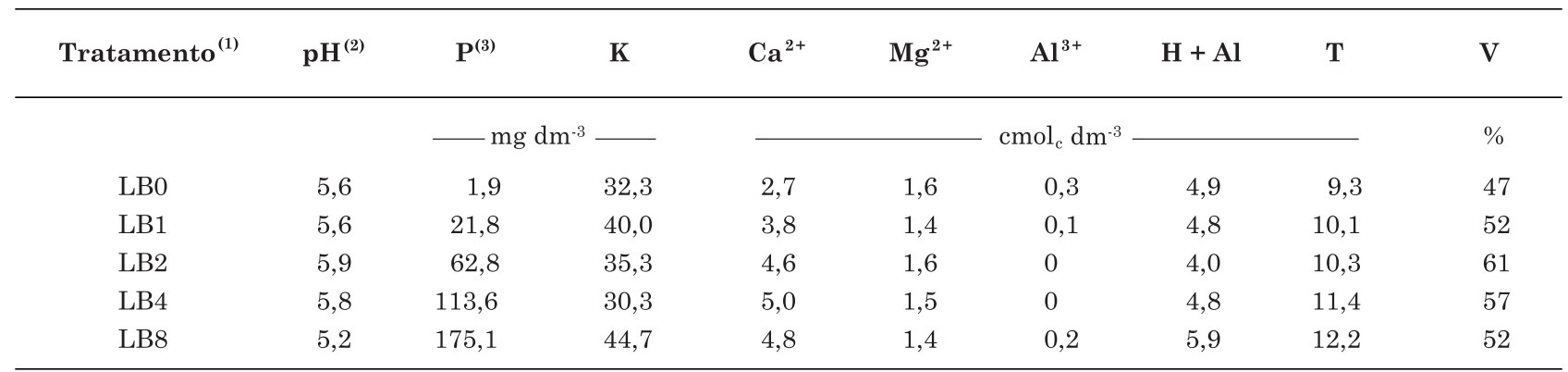

(1) LB0, LB1, LB2, LB4 e LB8: doses de lodo de esgoto visando suplir 0, 1, 2, 4 e 5 vezes a necessidade de $\mathrm{N}$ do milho. ${ }^{(2)} \mathrm{pH}$ em água: relação solo:água $(1: 2,5) .{ }^{(3)}$ P-Mehlich-1. 
Quadro 2. Quantidades de lodo de esgoto e $\mathrm{K}_{2} \mathrm{O}$ aplicadas nos seis cultivos de milho

\begin{tabular}{|c|c|c|c|c|c|c|c|c|c|c|c|c|c|}
\hline \multirow{3}{*}{ Tratamento $^{(1)}$} & \multicolumn{6}{|c|}{ Lodo de esgoto, base seca } & \multirow{3}{*}{ Total } & \multirow{2}{*}{\multicolumn{6}{|c|}{$\frac{\mathrm{K}_{2} \mathbf{O}^{(2)}}{\text { Cultivo }}$}} \\
\hline & \multicolumn{6}{|c|}{ Cultivo } & & & & & & & \\
\hline & $1^{o}$ & $2^{o}$ & $3^{o}$ & $4^{\circ}$ & $5^{\circ}$ & $6^{\circ}$ & & $1^{\circ}$ & $2^{\circ}$ & $3^{o}$ & $4^{\circ}$ & $5^{\circ}$ & $6^{\circ}$ \\
\hline & \multicolumn{7}{|c|}{$-\mathrm{tha} \mathrm{a}^{-1}$} & \multicolumn{6}{|c|}{ - $\mathrm{kg} \mathrm{ha}^{-1}$} \\
\hline LB0 & 0 & 0 & 0 & 0 & 0 & 0 & 0 & 0 & 0 & 0 & 0 & 0 & 0 \\
\hline LB1 & 8 & 4 & 5,6 & 5,6 & 3 & 3,8 & 30 & 3 & 28 & 40 & 87 & 57 & 36 \\
\hline BL2 & 16 & 8 & 10,9 & 10,9 & 6,5 & 7,7 & 60 & 0 & 19 & 8 & 69 & 45 & 21 \\
\hline LB4 & 32 & 16 & 21,5 & 21,5 & 13 & 16 & 120 & 0 & 4 & 0 & 33 & 21 & 0 \\
\hline LB8 & 64 & 32 & 43 & 43 & 26 & 32 & 240 & 0 & 0 & 0 & 0 & 0 & 0 \\
\hline
\end{tabular}

(1) LB0, LB1, LB2, LB4 e LB8: doses de lodo de esgoto visando suprir 0, 1, 2, 4 e 5 vezes a necessidade de N do milho. ${ }^{(2)}$ Quantidade de $\mathrm{K}_{2} \mathrm{O}$ utilizada para complementar o $\mathrm{K}$ fornecido pelo lodo de esgoto e para atender as exigências nutricionais do milho.

Quadro 3. Características químicas dos lotes de lodo de esgoto utilizados no tratamento do solo, em seis ciclos de cultivo de milho

\begin{tabular}{|c|c|c|c|c|c|c|c|}
\hline \multirow{2}{*}{ Atributo } & \multirow{2}{*}{ Unidade $^{(1)}$} & \multicolumn{6}{|c|}{ Cultivo de milho } \\
\hline & & $1^{\circ}$ & $2^{\circ}$ & $3^{\circ}$ & $4^{\circ}$ & $5^{\circ}$ & $6^{\circ}$ \\
\hline pH (água) & - & 6,6 & 6,4 & 6,4 & 8,5 & 8,0 & 8,3 \\
\hline Teor de água ${ }^{(2)}$ & $\%$ & 66,4 & 80,2 & 71,2 & 79,5 & 78,8 & 81,2 \\
\hline Sólidos voláteis & $\%$ & 43,0 & nd & 56,8 & 62,6 & 59,6 & 62,0 \\
\hline C orgânico & $\mathrm{g} \mathrm{kg}^{-1}$ & 248 & 271 & 293 & 354 & 534 & 312 \\
\hline $\mathrm{N}$ total $^{(2)}$ & $\mathrm{g} \mathrm{kg}^{-1}$ & 21 & 49,7 & 42,1 & 40,8 & 79,7 & 43,5 \\
\hline $\mathrm{N}$-amoniacal & $\mathrm{mg} \mathrm{kg}^{-1}$ & 1.567 & 1.156 & 2.402 & 2.095 & 2.301 & nd \\
\hline N-nítrico & $\mathrm{mg} \mathrm{kg}^{-1}$ & 106 & 106 & 51 & 15 & 23 & nd \\
\hline $\mathrm{P}$ & $\mathrm{g} \mathrm{kg}^{-1}$ & 15,9 & 31,2 & 26,9 & 17,7 & 17,9 & 16,1 \\
\hline $\mathrm{K}$ & $\mathrm{g} \mathrm{kg}^{-1}$ & 1,0 & 2,0 & 1,0 & 1,5 & 1,0 & 1,2 \\
\hline $\mathrm{Ca}$ & $\mathrm{g} \mathrm{kg}^{-1}$ & 40,3 & 22,8 & 47,8 & 20,1 & 19,4 & 13,4 \\
\hline $\mathrm{Mg}$ & $\mathrm{g} \mathrm{kg}^{-1}$ & 3,0 & 3,7 & 4,5 & 3,7 & 3,8 & 2,7 \\
\hline $\mathrm{Na}$ & $\mathrm{g} \mathrm{kg}^{-1}$ & 0,5 & 0,6 & 0,5 & 0,5 & 0,9 & 0,6 \\
\hline $\mathrm{S}$ & $\mathrm{g} \mathrm{kg}^{-1}$ & 13,4 & 10,8 & 17,1 & 11,7 & 14,5 & 12,6 \\
\hline $\mathrm{Fe}$ & $\mathrm{g} \mathrm{kg}^{-1}$ & 54,2 & 32,5 & 38,0 & 39,1 & 32,1 & 31,9 \\
\hline Mo & $\mathrm{mg} \mathrm{kg}^{-1}$ & $<0,01$ & $<1$ & $<0,01$ & $<0,01$ & $<0,1$ & $<0,01$ \\
\hline $\mathrm{Mn}$ & $\mathrm{mg} \mathrm{kg}^{-1}$ & 430 & 335 & 419 & 278 & 247 & 209 \\
\hline $\mathrm{Cu}$ & $\mathrm{mg} \mathrm{kg}^{-1}$ & 1.058 & 1.046 & 953 & 682 & 868 & 805 \\
\hline $\mathrm{Zn}$ & $\mathrm{mg} \mathrm{kg}^{-1}$ & 2.821 & 3.335 & 3.372 & 2.328 & 3.330 & 2.888 \\
\hline B & $\mathrm{mg} \mathrm{kg}^{-1}$ & 36,2 & 11,2 & 29,3 & 10,7 & 17,6 & 11,1 \\
\hline Cr total & $\mathrm{mg} \mathrm{kg}^{-1}$ & 823 & 1.071 & 1.297 & 609 & 640 & 700 \\
\hline $\mathrm{Ni}$ & $\mathrm{mg} \mathrm{kg}^{-1}$ & 518 & 483 & 606 & 331 & 270 & 253 \\
\hline $\mathrm{Al}$ & $\mathrm{mg} \mathrm{kg}^{-1}$ & 28,8 & 25,3 & 23,3 & 11,9 & 14,2 & 15,1 \\
\hline $\mathrm{Cd}$ & $\mathrm{mg} \mathrm{kg}^{-1}$ & 12,8 & 9,5 & 9,4 & 16,2 & 14,0 & 14,1 \\
\hline $\mathrm{Pb}$ & $\mathrm{mg} \mathrm{kg}^{-1}$ & 364 & 233 & 349 & 138 & 149 & 127 \\
\hline As & $\mathrm{mg} \mathrm{kg}^{-1}$ & $<1$ & $<1$ & $<1$ & $<0,01$ & $<0,1$ & $<0,01$ \\
\hline $\mathrm{Ag}$ & $\mathrm{mg} \mathrm{kg}^{-1}$ & $<0,01$ & $<1$ & $<0,01$ & $<0,01$ & $<0,1$ & $<0,01$ \\
\hline
\end{tabular}

(1) Valores dados em base seca, seguindo protocolo analítico da EPA SW-846-3051, descrito em EPA (1986). Nd: não determinado.

${ }^{(2)}$ Valores de umidade e $\mathrm{N}$ total determinados em amostras recebidas nas condições originais, na Embrapa Meio Ambiente. 
suprir a necessidade de N do milho; no LB2, aplicaramse, no solo, duas vezes a dose recomendada em LB1; no LB4, foi feita aplicação de quatro vezes a dose recomendada em $\mathrm{LB} 1$ e, no $\mathrm{LB} 8$, foi realizada a aplicação de oito vezes a dose de nitrogênio requerida pelo milho e recomendada em LB1. Como referência, foi amostrada uma área sob mata nas adjacências do local do experimento.

Os ácidos húmicos utilizados nesse experimento foram isolados de acordo com o procedimento sugerido pela Sociedade Internacional de Substâncias Húmicas (IHSS) (Swift, 1996; Machado, 1999), sendo realizado um pré-tratamento das amostras do solo com $\mathrm{HCl}$ $0,1 \mathrm{~mol} \mathrm{~L}^{-1}$ e a extração com $\mathrm{NaOH} 0,1 \mathrm{~mol} \mathrm{~L}^{-1}$, na razão solo:solvente de $1: 10(\mathrm{p}: \mathrm{v})$, em atmosfera inerte de $\mathrm{N}_{2}$. A fração de ácido húmico foi obtida com a diminuição do $\mathrm{pH}$ da solução até $1,5 \pm 0,5$, utilizandose $\mathrm{HCl} 6 \mathrm{~mol} \mathrm{~L}^{-1}$. A redissolução e precipitação foram repetidas três vezes. Após essa etapa, o ácido húmico precipitado foi purificado utilizando-se solução $\mathrm{HF}+\mathrm{HCl}$ (preparada com $5 \mathrm{~mL}$ de $\mathrm{HF} 48 \%$ e $5 \mathrm{~mL}$ de $\mathrm{HCl}$ concentrado, sendo o volume da solução completado para $1 \mathrm{~L}$ com água destilada), com a agitação por $12 \mathrm{~h}$ das amostras, que foram, em sequência, centrifugadas a $9.000 \mathrm{rpm}$ durante $10 \mathrm{~min}$, repetindo-se o processo por três vezes. Posteriormente, as amostras foram dialisadas em água deionizada, utilizando-se membranas com peso molecular de corte igual a $10.000 \mathrm{~g} \mathrm{~mol}^{-1}$. A diálise dos materiais foi encerrada quando a condutividade elétrica foi igual a da água utilizada na diálise. Em seguida, as amostras foram congeladas, liofilizadas e armazenadas em dessecador, até serem utilizadas nas análises de espectroscopia no infravermelho com transformada de Fourier (IVTF).

Os espectros foram obtidos utilizando-se o espectrofotômetro Excalibur FT 3000 Series detector DTGS, operando em um intervalo de comprimento de onda de 4.000 a $400 \mathrm{~cm}^{-1}$, resolução de $4 \mathrm{~cm}^{-1}$, dotado de um sistema computadorizado, com o software Digilab Merlin 3.3. As pastilhas de $\mathrm{KBr}$ foram obtidas aplicando-se uma pressão de $3 \mathrm{t} \mathrm{cm}^{-2}$, durante $1 \mathrm{~min}$, a uma mistura de $3 \mathrm{mg}$ de ácido húmico e $100 \mathrm{mg}$ de $\mathrm{KBr}$. Nessa etapa, foram analisadas também amostras do lodo de esgoto utilizado no experimento, seguindo o mesmo método descrito para os ácidos húmicos.

Após a obtenção dos espectros, foi determinado, para cada amostra, nos diferentes tratamentos, o índice de hidrofobicidade (IH), que foi obtido pela relação entre a intensidade da absorbância de $2.929 \mathrm{~cm}^{-1}$, correspondente ao estiramento $\mathrm{C}-\mathrm{H}$ do grupamento hidrofóbico $\mathrm{C}-\mathrm{H}_{3}$ alifático, com o teor de $\mathrm{C}$ na forma de fração ácido húmico (Dias et al., 2007) de cada tratamento testado, de acordo com Capriel et al. (1995). O índice de condensação (IC) foi determinado a partir de modificações feitas no método de cálculo proposto por Freixo et al., (2002), ou seja, calculou-se a relação entre intensidade de absorbância na regiões espectrais de 1.660 e $2.929 \mathrm{~cm}^{-1}$. A relação obtida foi dividida, a seguir, pelo teor de C-fração de ácido húmico de cada tratamento testado, tendo como bases os resultados apresentados por Dias et al. (2007). As intensidades de absorbâncias utilizadas nos cálculos descritos anteriormente foram obtidas em função da correção de linha de base espectral, visando normalizar todas as regiões do espectro de IV.

Os índices obtidos foram analisados estatisticamente por meio da análise de variância e as diferenças entre as médias foram avaliadas pelo teste de ScottKnott a $5 \%$, sendo utilizado nessa etapa o programa estatístico SISVAR (Ferreira, 2000).

\section{RESULTADOS E DISCUSSÃO}

Os espectros foram obtidos em triplicatas, sendo apresentado um espectro por tratamento, que foi analisado de acordo com as atribuições propostas por Stevenson (1994); Benites et al. (1999); Canellas (1999) e Silverstein \& Webster (2000). O espectro obtido para o lodo de esgoto (Figura 1) apresenta características diferenciadas das demais amostras, com picos bastante evidentes na região de $2.900 \mathrm{~cm}^{-1}$, que corresponde ao estiramento alifático de grupo C-H, e em $1.033 \mathrm{~cm}^{-1}$, que corresponde ao estiramento $\mathrm{C}-\mathrm{O}$ de polissacarídeos. Assim, se o uso de lodo de esgoto afetar a MOS, é possível que esse resíduo promova mudanças mais pronunciadas nessas regiões espectrais.

Os espectros obtidos para as amostras de ácido húmico extraídas de solos tratados com lodo de esgoto (Figura 2) podem ser classificados como do tipo III, de acordo com a classificação proposta por Stevenson (1994), em razão da presença de bandas características nos comprimentos $3.400 \mathrm{~cm}^{-1}, 2.900 \mathrm{~cm}^{-1}, 1.720 \mathrm{~cm}^{-1}$, $1.600 \mathrm{~cm}^{-1}, 1.200 \mathrm{~cm}^{-1} \mathrm{e} 1.540 \mathrm{~cm}^{-1}$.

Os espectros de infravermelho para os ácidos

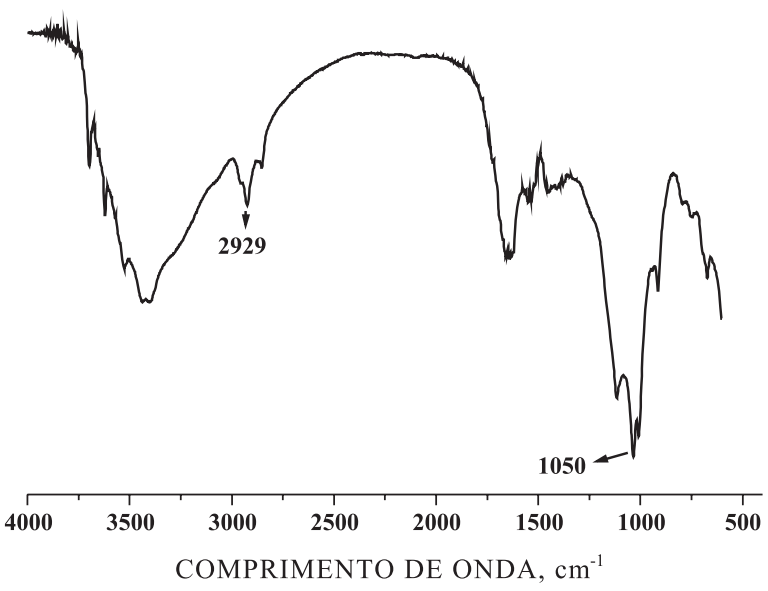

Figura 1. Espectro de infravermelho do lodo de esgoto utilizado no estudo. 
húmicos são apresentados na figura 2. A região de $3.750-3.400 \mathrm{~cm}^{-1}$ é característica de estiramento e deformações de ligações $\mathrm{O}-\mathrm{H}$ e Si-O, ou seja, caracterizada pela presença de impurezas minerais (Russel, 1987, citado por Bertoncini, 2002). Na faixa espectral de comprimentos de $3.440-3.300 \mathrm{~cm}^{-1}$, verificou-se a presença de uma banda de absorção larga para as amostras de ácido húmico, que se refere ao estiramento $\mathrm{COOH}$ em ponte de $\mathrm{H}$, de modo que a força das ligações intramoleculares indica a extensão do alargamento; quanto mais pontes, mais ácido é o material analisado e mais larga é a absorção nessa região. A presença de bandas de absorção referentes a grupos N-H: é característica dessa região. No entanto, neste estudo, não foi possível observar esse grupamento, devido à sobreposição de bandas nos espectros. Comparando os espectros obtidos para as amostras de ácido húmico, nessa faixa de comprimento de onda, em todos os tratamentos, não foi possível constatar diferenças, sendo uma característica similar entre os espectros a presença de uma larga banda de absorção. Segundo Freixo (2000), as ligações entre o grupamento $\mathrm{O}-\mathrm{H}$ são muito fortes (ligações de $\mathrm{H}^{+}$), com menor vibração das moléculas, que resulta em uma banda larga, quando as amostras são analisadas no infravermelho.

A banda de absorção característica dos grupos metil e metileno $\left(\mathrm{CH}_{2}\right.$ e $\left.\mathrm{CH}_{3}\right)$ ocorre em comprimento de onda de $2.940-2.840 \mathrm{~cm}^{-1}$. Analisando os espectros de ácido húmico, foi possível verificar que os tratamentos apresentaram, nessa faixa, bandas de absorção características de grupos $\mathrm{CH}_{2}$. A ocorrência de bandas de absorção entre 2.950 e $2.850 \mathrm{~cm}^{-1}$ são atribuídas ao estiramento C-H alifático (Dick et al., 2003).

Neste estudo, observou-se que, na maior dose acumulada de lodo (LB8), ocorre maior presença de compostos alifáticos nos ácidos húmicos, resultado que está de acordo com os encontrados por Bertoncini (2002), em estudo que avaliou o impacto da adição de biossólidos nas substâncias húmicas de Latossolo Vermelho Amarelo distrófico, em dose acumulada de lodo de $388 \mathrm{t} \mathrm{ha}^{-1}$ (base seca). Constatou-se, neste estudo, que as bandas de $\mathrm{C}$-H alifático foram mais intensas nos solos tratados com esse resíduo, e que a adição de biossólido enriquece, em termos absolutos, os ácidos húmicos em compostos alifáticos. A presença do grupamento $-\mathrm{CH}_{2}$ - é sempre acompanhada de picos característicos da deformação axial de C-H alifático na região de absorção de 1450-1460 $\mathrm{cm}^{-1}$. Em todos os espectros, nota-se uma absorção próxima a essa região, ratificando a presença de cadeias alifáticas nos ácidos húmicos. É possível identificar que essa banda é intensificada nas amostras extraídas dos solos onde foi feita aplicação do lodo de esgoto, se comparadas com as bandas obtidas dos solos sob mata e o que recebeu adubação NPK.

$\mathrm{Na}$ faixa de absorção de $2.500-2.600 \mathrm{~cm}^{-1}$, foi verificada uma banda de pouca intensidade correspondente ao estiramento axial de $\mathrm{OH}$ de grupo

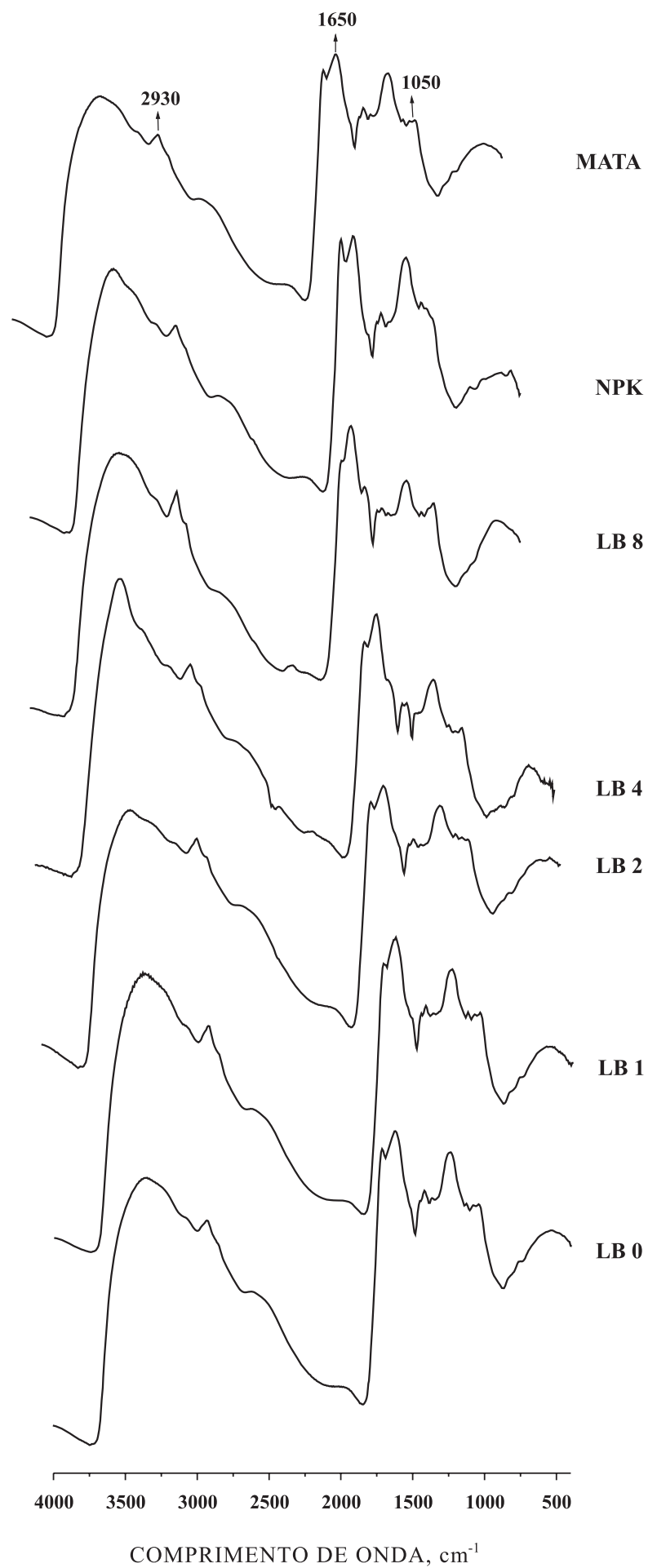

Figura 2. Espectros de infravermelho dos ácidos húmicos de Latossolo sob efeito de doses crescentes de lodo de esgoto; LB0, LB1, LB2, LB4 e $\operatorname{LB} 8(0,1,2,4$ e 5 vezes a necessidade de $\mathrm{N}$ do milho) e área sob mata e adubação mineral (NPK).

carboxílico. Nos espectros obtidos, foi possível observar a presença dessas bandas, em razão do processo de purificação das mesmas com HF, que possibilita o 
rompimento de ligações organometálicas, formando complexos solúveis com os minerais e tornando livres os grupos carboxílicos, o que favorece a sua identificação (Piccolo,1988).

A banda de absorção próxima de $1.710 \mathrm{~cm}^{-1}$ aparece em todos os espectros, sendo atribuída ao estiramento $\mathrm{C}=\mathrm{O}$ de $\mathrm{COOH}$, aos aldeídos e às cetonas. Sua intensidade foi pouco pronunciada entre os ácidos húmicos de diferentes tratamentos. Os espectros apresentam também uma pequena banda de absorção na região de $1.630-1.660 \mathrm{~cm}^{-1}$, característico de estiramento $\mathrm{C}=\mathrm{O}$ de grupo amina, quinona e, ou, de bandas conjugadas de $\mathrm{C}=\mathrm{O}$ de cetonas, não sendo identificadas diferenças nos espectros, em todos os tratamentos, para os ácidos húmicos.

$\mathrm{Na}$ região de $1.600-1.620 \mathrm{~cm}^{-1}$, que representa vibração do estiramento $\mathrm{C}=\mathrm{C}$ de alcenos conjugados, COO- e $\mathrm{C}=\mathrm{O}$ de quinonas (Canellas \& Rumjanek, 2005), foram observadas bandas espectrais que ocorreram também com a mesma intensidade em todos os tratamentos analisados no ácido húmico. A pequena banda de absorção na zona de $1.510-1.540 \mathrm{~cm}^{-1}$ pode estar relacionada ao estiramento $\mathrm{C}=\mathrm{C}$ aromático, cuja deformação ocorre de $800 \mathrm{~cm}^{-1}$ para baixo, podendo sugerir também a presença de amidas (Canellas, 1999).

Segundo Stevenson (1994), as bandas de absorção entre $1.400-1.390 \mathrm{~cm}^{-1}$ podem ser características de deformação $\mathrm{OH}$, estiramento de fenóis $\mathrm{OH}$, deformações de grupos $\mathrm{CH}_{2}$ e $\mathrm{CH}_{3}$ e ou estiramento antissimétrico de COO- Nessa região espectral foram observadas bandas de absorção referentes a esses grupamentos, entretanto, não foi possível verificar diferenças entre os tratamentos.

No comprimento de onda 1.280 a $1.200 \mathrm{~cm}^{-1}$ surgem bandas de absorção características de estiramento $\mathrm{C}-\mathrm{O}$ e deformações $\mathrm{OH}$ de $\mathrm{COOH}$, estiramento C-O-C de aril éter e fenóis. De acordo com Piccolo \& Stevenson (1982), a conversão de COOH para $\mathrm{COO}^{-}$causa a perda de absorção na faixa próxima a $1.230 \mathrm{~cm}^{-1}$, sugerindo uma possível explicação para a complexação com metais. Analisando os espectros (Figura 2), observa-se que as bandas de absorção foram mais intensas nas amostras de NPK, mata, LB0, LB1 e LB2, mostrando-se com menor intensidade para LB4 e LB8, sendo uma possível explicação para a alta concentração de $\mathrm{Fe}$ encontrada no solo avaliado, o que favorece a formação de complexos com o grupamento COO . Na região de 1.100-1.000 $\mathrm{cm}^{-1}$, surgem picos característicos de polissacarídeos nos ácidos húmicos, que foram menos evidentes na amostra que recebeu a maior dose de lodo de esgoto (LB8).

Os índices de hidrofobicidade e de condensação para as amostras de ácidos húmicos estudadas são apresentados no quadro 4 . Os IHs verificados neste estudo encontram-se dentro da faixa de variação daqueles valores observados para ácidos húmicos obtidos em estudo que testou o efeito de diferentes sistemas de rotação de culturas e preparo do solo sobre a matéria orgânica (Dick et al., 2003), e são similares aos valores de IH obtidos para amostras de fração leve livre e leve intra-agregados (Freixo et al., 2002). Foram observadas diferenças significativas para o IH entre os diferentes tratamentos testados, sendo observados menores valores de IH para as áreas adubadas com as maiores doses de lodo de esgoto, ou seja, para os tratamentos LB4 e LB8. Em mesma área experimental deste estudo, ao utilizar a técnica de ressonância paramagnética eletrônica para avaliar o efeito da aplicação continuada de lodo sobre a natureza química de ácidos húmicos, González-Pérez et al. (2006) verificaram que o uso frequente de elevadas doses de lodo, em comparação às menores, diminuiu a concentração de radicais livres do tipo semiquinona (RLS) na MOS. Nesse mesmo estudo, a área de controle sob vegetação natural e as cultivadas com baixas doses de lodo foram as que apresentaram as maiores concentrações de RLS, o que indica maior grau de humificação dos ácidos húmicos, já que esse atributo pode ser considerado como um indicativo do grau de humificação da MOS. Segundo GonzálezPérez et al. (2006), o menor grau de humificação do AHs oriundos de áreas com maior fertilização de lodo pode ser atribuído à menor carga de compostos aromáticos na matéria orgânica das partidas de lodo aplicadas no solo. Os dados verificados por GonzálezPéres et al. (2006) estão de acordo com os observados neste estudo, contudo discordam dos obtidos por Marques (1998), que observou maiores níveis de RLS e grau de humificação de ácidos húmicos extraídos de Latossolo adubado com lodo. No estudo conduzido por Adani \& Tombone (2005), a natureza química de ácidos húmicos extraídos de solo adubado com lodo de

Quadro 4. Índices de hidrofobicidade (IH) e de condensação (IC) de ácidos húmicos extraídos de Latossolo $(0-10 \mathrm{~cm})$ adubado com doses crescentes de lodo de esgoto de Barueri (LB)

\begin{tabular}{ccc}
\hline & IH $^{(2)}$ & IC $^{(2)}$ \\
Tratamento $^{(\mathbf{1})}$ & $0,63(0,10) \mathrm{b}$ & $1,45(0,27) \mathrm{a}$ \\
LB0 & $0,65(0,18) \mathrm{b}$ & $0,66(0,35) \mathrm{b}$ \\
LB1 & $0,56(0,07) \mathrm{b}$ & $0,70(0,18) \mathrm{b}$ \\
LB2 & $0,38(0,05) \mathrm{c}$ & $0,44(0,19) \mathrm{c}$ \\
LB4 & $0,42(0,24) \mathrm{c}$ & $0,46(0,14) \mathrm{c}$ \\
LB8 & $1,08(0,01) \mathrm{a}$ & $0,49(0,04) \mathrm{c}$ \\
Mata & $0,79(0,25) \mathrm{b}$ & $0,78(0,48) \mathrm{b}$ \\
NPK & & \\
\end{tabular}

(1) LB0, LB1, LB2, LB4 e LB8 (doses de lodo de esgoto visando suprir $0,1,2,4$ e 5 vezes a necessidade de $\mathrm{N}$ do milho); NPK: (adubação mineral com formulado NPK); ${ }^{(2)}$ Valores entre parênteses referem-se ao desvio-padrão da média. Médias seguidas de mesma letra não diferiram entre si pelo teste de ScottKnott a 5\%. IH: (Intensidade de absorbância na região do Califático-2.929 $\mathrm{cm}^{-1} / \mathrm{C}$-fração ácido húmico); IC: (C-absorbância em $1.660 \mathrm{~cm}^{-1} / \mathrm{C}$-absorbância em $\left.2.929 \mathrm{~cm}^{-1}\right) / \mathrm{C}$-fração ácido húmico. 
esgoto refletiu o enriquecimento de materiais recalcitrantes nos ácidos húmicos do lodo. Esses efeitos contratantes de aplicações de lodo de esgoto sobre a matéria orgânica do solo podem estar associados, entre outros fatores, a condições climáticas, gênese e origem do solo, doses e natureza química do resíduo aplicado e métodos de compostagem do resíduo (Adani \& Tambone, 2005; González-Pérez et al., 2006).

Segundo Capriel et al. (1995), quanto menor o IH, mais reduzida é a concentração de C-alifático e menos hidrofóbico é o acido húmico analisado. Tendo em vista que os grupos alifáticos de $\mathrm{C}$ são consideradas uma das estruturas mais recalcitrantes da matéria orgânica do solo (Baldock et al., 1997), torna-se possível, a partir desse atributo, inferir o grau de humificação da MOS (Dick et al., 2003). De fato, cada tipo de molécula em resíduos decompostos apresenta um grau característico de recalcitrância bioquímica, que é definida pela força de ligações inter e intramoleculares, pelo grau de polimerização, pela regularidade das unidades estruturais nos polímeros e pelo teor de grupos funcionais aromáticos e alifáticos na fração orgânica analisada (Baldock et al., 1997; Gleixner et al., 2001; Baldock et al., 2004). Segundo Zech et al. (1997), em solos tropicais, a dominância na matéria orgânica do solo de constituintes aromáticos é o fator responsável pela maior estabilidade no ambiente das frações orgânicas.

Assim, em função dos resultados observados, a aplicação das maiores doses de lodo de esgoto implica menor presença em amostras de ácidos húmicos de estruturas orgânicas mais recalcitrantes, portanto em maior biodisponibilidade do material húmico.

O índice de condensação (IC) é utilizado na avaliação do grau de condensação/humificação da molécula (Freixo et al., 2002), de modo que, com esta perspectiva, foram obtidos, neste estudo, ICs variando na faixa de 0,44 a 1,45 , com os maiores valores observados nas áreas sem aplicação de lodo ou com a adição de formulado NPK. O uso de doses elevadas de lodo (LB4 e LB8) resultou nos menores valores de IC, cujos ácidos húmicos são similares, em constituição química, ao material húmico oriundo da área de mata. Nas áreas que receberam menores doses de lodo (L1 e LB2), os valores de IC foram similares aos observados para os tratamentos LB0 e NPK. Na área de mata, a fração de ácido húmico é enriquecida em C-alifático (maior IH), contudo não se trata de matéria orgânica com alto grau de condensação, em razão do baixo valor de IC medido.

Com base nas considerações feitas por Freixo et al. (2002), pode-se inferir que as áreas que receberam elevadas doses de lodo de esgoto são as que apresentam menores teores de materiais orgânicos menos enriquecidos em materiais recalcitrantes. A matéria orgânica do solo com estruturas mais humificadas, em detrimento das mais lábeis, implica diminuição da atividade microbiana e menor agregação do solo (Capriel et al., 1995). Entretanto, o maior aporte no solo de substâncias húmicas hidrofóbicas pode representar um mecanismo importante de sequestro de $\mathrm{C}$ no solo, contribuindo, assim, para mitigar as emissões de $\mathrm{CO}_{2}$ de solos agrícolas (Spaccini et al., 2002), embora a capacidade de retenção de água do solo possa ser comprometida pela baixa disponibilidade de frações orgânicas hidrofílicas (Ellerbrock et al., 2005).

Em relação à matéria orgânica, o manejo adequado do solo deve proporcionar um equilíbrio de frações mais e menos lábeis, no sentido de que sejam asseguradas condições para o pleno crescimento das culturas, ou seja, maior disponibilidade de nutrientes, maior densidade de cargas no solo, maior retenção de água, maior agregação das partículas do solo, maior atividade microbiana etc. Nesse sentido, o ácido húmico da área de mata sinaliza a ocorrência dessas condições, tendo em vista que o enriquecimento de C-alifático nessas frações e o menor grau de condensação parecem refletir o equilíbro de frações mais e menos lábeis em solo. A aplicação de lodo, principalmente em doses mais elevadas, parece assegurar um aumento da biodisponibilidade das frações orgânicas no solo, o que está de acordo com os dados apresentados por Fernandes et al. (2005), que verificaram aumentos da respiração basal, dos teores de $\mathrm{C}$ e $\mathrm{N}$ na biomassa, da atividade enzimática e do quociente metabólico microbiano proporcionais à adição de doses de lodo de esgoto ao solo.

\section{CONCLUSÕES}

1. Os ácidos húmicos, quanto à natureza química, apresentam grupos aromáticos, $-\mathrm{OH}$ fenólicos, $-\mathrm{COOH}$, estruturas alifáticas, carboidratos e impurezas minerais.

2. As amostras de ácido húmico oriundas das áreas com aplicações continuadas de elevadas doses de lodo de esgoto são as que apresentam menor concentração de C-alifático (menor índice de hidrofobicidade) e menor grau de condensação (menor índice de condensação). A ausência de aplicação de lodo ou o uso exclusivo de formulado NPKimplica frações orgânicas no solo mais ricas em estruturas recalcitrantes e com maior grau de condensação. As amostras de ácidos húmicos originadas da área de mata são ricas em C-alifático, entretanto apresentam baixo grau de condensação.

\section{AGRADECIMENTOS}

À equipe de apoio da Embrapa Meio Ambiente, pela contribuição na elaboração, manutenção e condução do experimento. Ao Conselho Nacional de Desenvolvimento Científico e Tecnológico (CNPq), pelo financiamento do projeto de pesquisa (Processo 479565/ 2003-1) e pela concessão da bolsa de estudo. Ao prof. 
Luciano P. Canellas (UENF), pelas valiosas sugestões para a execução das análises laboratoriais, interpretação dos resultados e correções de aproximações deste estudo.

\section{LITERATURA CITADA}

ADANI, F. \& TAMBONE, F. Long-term effect of sewage sludge application on soil humic acids. Chemosphere, 60:12141221,2005

BALDOCK, J.A.; MASIELLO, C.A.; GÉLINAS, Y. \& HEDGES, J.I. Cycling and composition of organic matter in terrestrial and marine ecosystems. Marine Chem., 92:39-64, 2004.

BALDOCK, J.A.; OADES, J.M.; NELSON, P.N.; SKENE, T.M.; GOLCHIN, A. \& CLARKE, P. Assessing the extent of decomposition of natural organic materials using soilstate 13C NMR spectroscopy. Aust. J. Soil Res., 35:1061$1083,1997$.

BENITES, V.M.; MENDONÇA, E.S.; SCHAEFER, C.E.R. \& MARTIN NETO, L. Caracterização dos ácidos húmicos extraídos de um Latossolo vermelho-amarelo e de um Podzol por análise termodiferencial e pela espectroscopia de absorção no infravermelho. R. Bras. Ci. Solo, 23:543$551,1999$.

BERTONCINI, E.I. Comportamento de $\mathrm{Cd}, \mathrm{Cr}, \mathrm{Cu}, \mathrm{Ni}$ e $\mathrm{Zn}$ em latossolos sucessivamente tratados com biossólidos: Extração sequencial, fitodisponibilidade e caracterização de substâncias húmicas. Piracicaba, Escola Superior de Agricultura Luiz de Queiroz, 2002. 191p. (Tese de Doutorado)

CANELLAS, L.P. \& RUMJANEK, V.M. Espectroscopia na região do infravermelho. In: CANELLAS, L.P. \& SANTOS, G. Humosfera: Tratado preliminar sobre a química das substâncias húmicas. Campos dos Goytacazes, Universidade Estadual do Norte Fluminense, 2005. p.143-159.

CANELLAS, L.P.; SANTOS, G.A.; RUMJANEK, V.M.; MORAES, A.A. \& GURIDI, F. Avaliação de características de ácidos húmicos de resíduos sólidos urbanos I. Métodos espectroscópicos (IV, UV e RMN ${ }^{13} \mathrm{C}$ ) e microscopia eletrônica de varredura. R. Bras. Ci. Solo, 24:741-750, 2000.

CANELLAS, L.P. Avaliação de características físico-químicas de ácidos húmicos. Seropédica, Universidade Federal Rural do Rio de Janeiro, 1999. 162p. (Tese de Doutorado).

CAPRIEL, P.; BECK, T.; BORCHERT, H.; GRONHOLZ, J. \& ZACHMANN, G. Hydrophobicity of the organic matter in arable soils. Soil Biol. Biochem., 27:1453-1458, 1995.

DIAS, B. O.; SILVA, C.A.; SOARES, E.M.B. \& BETTIOL, W. Estoque de carbono e quantificação de substâncias húmicas em Latossolo submetido à aplicação contínua de lodo de esgoto. R. Bras. Ci. Solo, 31:701-711, 2007.

DICK, D.P.; SANTOS, J.H.Z. \& FERRANTI, E.M. Chemical characterization and infrared spectroscopy of soil organic matter from two Southern Brazilian soils. R. Bras. Ci. Solo, 27:29-39, 2003.
ELLERBROCK, R.H.; GERKE, H.H.; BACHMANN, J. \& GOEBEL, M.O. Composition of organic matter fractions for explaining wettability of three forest soils. Soil Sci. Am. J., 69:57-66, 2005.

FERNANDES, S.A.P.; BETTIOL, W. \& CERRI, C.C. Effect of sewage sludge on microbial biomass, basal respiration, metabolic quotient and soil enzymatic activity. App. Soil Ecol., 30:65-77, 2005.

FERREIRA, D.F. Análises estatísticas por meio do Sisvar para Windows 4. 0. In: REUNIÃO ANUAL DA REGIÃO BRASILEIRA DA SOCIEDADE INTERNACIONAL DE BIOMETRIA, 45., São Carlos, 2000. Anais. São Carlos, Universidade Federal de São Carlos, 2000. p.255-258.

FREIXO, A.A.; CANELLA, L.P. \& MACHADO, P.L.O.A. Propriedades espectrais da matéria orgânica leve-livre e leve-intra-agregado de dois Latossolos sob plantio direto e preparo convencional. R. Bras. Ci. Solo, 26:445-453, 2002.

FREIXO, A.A. Caracterização da matéria orgânica de latossolo sob diferentes sistemas de cultivo através de fracionamento físico e espectroscopia de infravermelho. Seropédica, Universidade Federal Rural do Rio de Janeiro, 2000. 86p. (Tese de Mestrado)

GLEIXNER, G.; CZIMEZIK, C.J.; KRAMER, C.; LUEHKER, B. \& SCHMIDT, M.W.I. Plant compounds and their turnover and stabilization as soil organic matter. In: SHULZE, E.D.; HEIMANN, M.; HARRISON, S.; HOLLAND, E.; LLOYD, J.; PRENTICE, I.C. \& SCHIMEL, D., eds. Global biogeochemical cycles in the climate system. San Diego, Academic Press, 2001. p.201-215.

GONZÁLEZ-PÉREZ, M.; MARTIN-NETO, L.; COLNAGO, L.A.; MILORI, D.M.B.P.; CAMARGO, AO.; BERTON, R. \& BETTIOL, W. Characterization of humic acids extracted from sewage sludge-amended Oxisols by electron paramagnetic resonance. Soil Till. Res., 91:95-100, 2006.

LABRADOR, J. La matéria orgânica em los agroecosistemas. Madrid, Ministério da Agricultura, Pesca y Alimentación, 1996. 174p.

MACHADO, P.L.O. Método para a extração de substâncias húmicas do solo: Ácido húmico e ácido fúlvico. Rio de Janeiro, Embrapa Solos, 1999. 4p. (Comunicado Técnico, 1)

MARQUES, J.C. Caracterização química e espectroscópica de substâncias húmicas de solos submetidos à adição de lodo de esgoto. São Carlos, Universidade Federal de São Carlos, 1998. 93p. (Tese de Mestrado)

OLIVEIRA, F.C.; MATTIAZZO, M.E.; MARCIANO, C.R. \& ROSSETO, R. Efeitos de aplicações sucessivas de lodo de esgoto em um Latossolo Amarelo distrófico cultivado com cana-de-açúcar: Carbono orgânico, condutividade elétrica, pH e CTC. R. Bras. Ci. Solo, 26:505-519, 2002.

PICCOLO, A. Characterization of soil humic extracts obtained by some organic and inorganic solvents and purified by HCl-HF treatment. Soil Sci., 146:418-426, 1988.

PICCOLO, A. \& STEVENSON, F.J. Infrared spectra of $\mathrm{Cu}^{2+}$, $\mathrm{Pb}^{2+}$ and $\mathrm{Ca}^{2+}$ complexes of soil humic substances. Geoderma, 27:195-208, 1982. 
ROCHA, G.N.; GONÇALVES, J.L.M. \& MOURA, I.M. Mudanças da fertilidade do solo e crescimento de um povoamento de Eucalyptus grandis fertilizado com biossólido. R. Bras. Ci. Solo, 28:623-639, 2004.

ROVIRA, S.P.A.; BRUNETTI, G.; POLO, A. \& SENESI, N. Comparative chemical and spectroscopic characterization of humic acids from sewage sludges and sludge-amended soils. Soil Sci., 167:235-245, 2002.

SENESI, N.; MIANO, T.M. \& BRUNETTI, G. Humic-like substances in organic amendments and effects on native soil humic substances. In: PICCOLO, A. Humic substances in terrestrial ecosystems. Amsterdan, Elsevier, 1996. p.531-593.

SILVERSTEIN, R.M. \& WEBSTER, F.X. Identificação espectrométrica de compostos orgânicos. Rio de Janeiro, Livros Técnicos e Científicos, 2000. 460p.

SIMONETE, M.A.; KIEHL, J.C.; ANDRADE, C.A. \& TEXEIRA, C.A.F. Efeito do lodo de esgoto em um argissolo e no crescimento e nutrição de milho. Pesq. Agropec. Bras., 38:1187-1195, 2003.
SONG, G.; NOVOTNY, E.H.; SIMPSON, A.J.; CLAPP, C.E. \& HAYES, H.B. Sequential exhaustive extraction of a Mollisol soil, and characterizations of humic components, including humin, by solid and solution state NMR. Eur. J. Soil Sci., 59:505-516, 2008.

SPACCINI, R.; PICCOLO, A.; CONTE, P.; HABERHAUER, G. \& GERZABEK, M.H. Increased soil organic carbon sequestration through hydrophobic protection by humic substances. Soil Biol. Biochem., 34:1839-1851, 2002.

STEVENSON. F.J. Humus chemistry: Genesis, composition, reactions. New York, J. Wiley \& Sons, 1982. 443p.

STEVENSON, F.J. Humus chemistry: Genesis, composition, reactions. New York, J. Wiley \& Sons, 1994. 496p.

SWIFT, R.S. Organic matter characterization. In: SPARKS, D., ed. Methods of soil analysis. Part 3: Chemical methods. Madison, SSSA, 1996. p.1011-1069. (Book Series, 5)

ZECH, W.; SENESI, N.; GUGGENBERGER, G.; KAISER, K.; LEHMANN, J.; MIANO, T.M.; MILTNER, A. \& SCHROTH, G. Factors controlling humification and mineralization of soil organic matter in the tropics. Geoderma, 79:117-161, 1997. 